\title{
Role of quantum entanglement due to a magnetic impurity on current magnification effect in mesoscopic open rings
}

\author{
Colin Benjamint, Sandeep K. Joshit, Debendranath Sahod甘 and A. M. Jayannavar $₫$ \\ Institute of Physics, Sachivalaya Marg, Bhubaneswar 751 005, Orissa, India
}

(October 22, 2018)

\begin{abstract}
We study the current magnification effect in presence of exchange scattering of electron from a magnetic impurity placed in one arm of an open mesoscopic ring. The exchange interaction causes entanglement of electron spin and impurity spin. Earlier studies have shown that such an entanglement causes reduction or loss of interference in the Aharonov-Bohm oscillations leading to decoherence. We find however, that this entanglement, in contradiction to the naive expectation of a reduction of current magnification, leads to enhancement as well as suppression of the effect. We also observe additional novel features like new resonances and current reversals.
\end{abstract}

PACS Nos.: 73.23.-b, 5.60.Gg, 72.10.Bg, 72.25.-b

Research on transport in mesoscopic open rings has provided several counterintuitive results 10 . The classic example of one such result is the gbservation of normal state Aharonov-Bohm (AB) effect A. Among other such effects in the context of mesoscopic systems, attention may be drawn upon the current amplification effect in the open ring system 8 wherein a current flowing in the lead is amplified, after passage through the junction, in one of the ring arms and appears as a negative current in the other ring arm. Like $\mathrm{AB}$ effect, this effect too is purely quantum mechanical in origin. This effect has been extended to thermal currents 11 and to spin currents in the presence of Aharonov-Casher flux 12 .

Recently we have studied the effect of decoherence on $\mathrm{AB}$ oscillations in mesoscopic open ring13. In the simple model that we consider, a magnetic impurity in one arm of the ring is coupled via exchange interaction to the incoming electron. Though the interaction induces spinflip scattering there is no exchange of energy. This fact along with entanglement 44 shows that spin-flip scattering arising due to exchange coupling with magnetic impurity reduces the $\mathrm{AB}$ oscillations and leads to decoherence 13 . The study of decoherence is important to understand the evolution of quantum system towards a classical one (destruction of entanglement due to environment). It is worthwhile to note that coupling to a single impurity or single degfae of freedom is enough to suppress the quantum effect 15 . In our case the impurity does not dynamics of its own. Generally, it is believed that only coupling with infinite degrees of freedom (a thermal bath) could induce dephasing. We know that the current magnification effect, to be discussed below, is a quantum effect. Hence one would like to study whether quantum entan- glement arising due to spin-impurity coupling can reduce this effect. The answer seems to be no, and the effect depends sensitively on the details of the geometry and other physical parameters of the problem.

In the case of a mesoscopic loop with unequal arms connected to two electron reservoirs at chemical potentials $\mu_{1}$ and $\mu_{2}$ via ideal leads currents $I_{\text {low }}$ and $I_{u p}$ flow in the lower and upper arm of the loop such that total current $I=I_{\text {low }}+I_{u p}$ is conserved in accordance with Kirchoff's law. In general these two currents differ in magnitude and are individually smaller than the total current $I$. However, in certain range of Fermi energies the current $I_{\text {low }}$ or $I_{\text {up }}$ may become larger than the total current $I$. This is the current magnification effect. To conserve the total current at the junctions, the current in the other arm becomes negative i.e., flows against the applied external field. This negative current continues to flow in the loop as a circulating current. The magnitude of the circulating current is the same as that of the negative current. The circulating current here arises in the absence of magnetic field. The effect of a simple impurity (an impurity without any internal structure) in one arm of the ring on the current magnification property has been studied earliert. It was found that the impurity could not only suppress but also enhance the current magnification depending on the Fermi energy. Thus the impurity plays a dual role as far as current magnification is concerned. The experimental possibility of the observation of this current magnification effect by looking at the orbital magnetic response of the loop is mentioned in Refs. 7,8,8.

In the following we study the current magnification effect in presence of a magnetic impurity in one arm of 
the ring. Consider a single channel loop of circumference $L$ coupled to two electron reservoirs by two ideal leads (see Fig. 1). We introduce a magnetic impurity atom (referred to as spin-flipper, or the flipper, for short) in one arm (upper) of the ring at length $l_{3}$ from junction $\mathrm{J} 1$ and $l_{4}$ from J2. The lower arm is of length $l_{2}$ and $L=l_{2}+l_{3}+l_{4}$. The spin of the electron $(\vec{\sigma})$ is thus coupled to the spin of the flipper $(\vec{S})$ via the exchange interaction $-J \vec{\sigma} \cdot \vec{S} \delta\left(x-l_{3}\right)$. This leads to scattering of the electron in which both the spin states of the electron and the impurity could change without any exchange of energy. This interaction conserves the total spin $(\vec{\sigma}+\vec{S})$ as well as the $z$-component of the total spin. The two reservoirs are kept at chemical potentials $\mu_{1}$ and $\mu_{2}$ respectively. When $\mu_{1}$ is greater than $\mu_{2}$, a net current flows from reservoir 1 on the left to the reservoir 2 on the right. Details of the ring geometry with the impurity are indicated in Fig. 1.

We follow the standard quantum waveguide theory $1 \mathrm{~B} 1 \mathrm{t}$ to study this problem. The wavefunctions $\left(\psi^{\prime} s\right)$ for the individual segments, as indicated in Fig. 1, can be written as below

$$
\begin{aligned}
\psi_{1}= & \left(e^{i k x}+r_{u} e^{-i k x}\right) \chi_{m} \alpha+ \\
& r_{d} e^{-i k x} \chi_{m+1} \beta, \\
\psi_{2}= & \left(A_{u} e^{i k x}+B_{u} e^{-i k x}\right) \chi_{m} \alpha+ \\
& \left(A_{d} e^{i k x}+B_{d} e^{-i k x}\right) \chi_{m+1} \beta, \\
\psi_{3}= & \left(C_{u} e^{i k x}+D_{u} e^{-i k x}\right) \chi_{m} \alpha+ \\
& \left(C_{d} e^{i k x}+D_{d} e^{-i k x}\right) \chi_{m+1} \beta, \\
\psi_{4}= & \left(E_{u} e^{i k x}+F_{u} e^{-i k x}\right) \chi_{m} \alpha+ \\
& \left(E_{d} e^{i k x}+F_{d} e^{-i k x}\right) \chi_{m+1} \beta, \\
\psi_{5}= & t_{u} e^{i k x} \chi_{m} \alpha+t_{d} e^{i k x} \chi_{m+1} \beta .
\end{aligned}
$$

where, $k$ is the wave-vector of incident electron. The subscripts $u$ and $d$ represent "up" and "down" spin states of the electron with the corresponding spinors $\alpha$ and $\beta$ respectively (i.e., $\sigma_{z} \alpha=\frac{1}{2} \alpha, \sigma_{z} \beta=-\frac{1}{\varepsilon} \beta$ ) and $\chi_{m}$ denotes the wave function of the impurity 17 with $S_{z}=m$ (i.e., $S_{z} \chi_{m}=m \chi_{m}$ ). The wavefunctions in equation (1) is a correlated function of the electron and impurity spins which takes into account the fact that the exchange interaction conserves the $z$-component of the total spin $(\vec{\sigma}+\vec{S})$. The incident electron is taken to be spin-up polarized. The reflected (transmitted) waves have amplitudes $r_{u}\left(t_{u}\right)$ and $r_{d}\left(t_{d}\right)$ corresponding to the "up" and "down" spin components respectively. Contimuity of the wave functions and the current conservation 610 , 17 at the junctions J1 and J2 imply the following boundary conditions.

$$
\begin{array}{r}
\psi_{1}(x=0)=\psi_{2}(x=0)=\psi_{3}(x=0), \\
\psi_{1}^{\prime}(x=0)=\psi_{2}^{\prime}(x=0)+\psi_{3}^{\prime}(x=0), \\
\psi_{4}^{\prime}\left(x=l_{3}\right)-\psi_{3}^{\prime}\left(x=l_{3}\right)=G(\vec{\sigma} \cdot \vec{S}) \psi_{3}\left(x=l_{3}\right), \\
\psi_{3}\left(x=l_{3}\right)=\psi_{4}\left(x=l_{3}\right), \\
\psi_{4}\left(x=l_{3}+l_{4}\right)=\psi_{5}(x=0)=\psi_{2}\left(x=l_{2}\right),
\end{array}
$$

$$
\psi_{2}^{\prime}\left(x=l_{2}\right)+\psi_{4}^{\prime}\left(x=l_{3}+l_{4}\right)=\psi_{5}^{\prime}(x=0) .
$$

Here $G=2 m J / \hbar^{2}$ is the dimensionless coupling constant indicative of the "strength" of the spin-exchange interaction. The primes denote the spatial derivatives of the wave functions. Equations (1) along with the boundary conditions (2) were solved to obtain the amplitudes, with these we evaluate the current densities in each arm of the ring. We have taken the flipper to be a spin-half object $(M=2)$ situated symmetrically at the center of the upper arm, i.e., we consider the case in which $l_{3}=l_{4}$. Now, depending upon the initial state of the flipper we have possibility of either spin-flip scattering $\left(\sigma_{z}=1 / 2, S_{z}=-1 / 2\right)$ or no spin-flip scattering $\left(\sigma_{z}=1 / 2, S_{z}=1 / 2\right)$, as demanded by the conservation of the total spin and its $z$-component. In the former case the problem reduces to that of a simplepotential scattering from the impurity as studied earlier $\mathrm{a}$.

We have set $\hbar=m=1$ and the value of interaction strength $G$ is given in dimensionless units throughout. The dimensionless current density $I_{2}$ in the small energy interval $d E$ (see Refs. 7,8) flowing in lower arm of the ring is defined as $I_{2}=I_{2 u}+I_{2 d}$, wherein $I_{2 u}=\left|A_{u}\right|^{2}-\left|B_{u}\right|^{2}$ and $I_{2 d}=\left|A_{d}\right|^{2}-\left|B_{d}\right|^{2}$. The current densities $I_{3}$ and $I_{4}$ flowing in the upper arm are similarly defined. Current conservation at the impurity site demands $I_{3}=I_{4}$ which we have verified. The total current flowing through the arms can be calculated by integrating the corresponding current densities over the Fermi energy interval $\mu_{1}$ to $\mu_{2}$ 요. In the present work we confine ourselves to the effect of the flipper on the circulating current density $I_{c}$. We have studied the behaviour of the current densities $I_{\text {low }}=I_{2}$ and $I_{u p}=I_{3}$ in the lower and the upper arms of the ring, as a function of $k L$. In any range of Fermi energy, if any one of these is negative, the magnitude of the negative current density capbe identified with that of the circulating current density $I_{-} I_{c}$. When $I_{\text {low }}$ is negative the direction of the circulating current density is clockwise and when $I_{u p}$ is negative then it is anti-clockwise. A clockwise circulating current density is taken to be positive and an anti-clockwise one, negative according to the usual convention. It has been seen earlier that there is an enhancement in current magnification in presence of a simple impurity(no spin-flip) 6 . In the following paragraphs we describe our results graphically.

Figure 2 shows the plot of circulating current density $\left(I_{c}\right)$ versus $k L$ for the two separate cases of spin-flip scattering and no-spin-flip scattering. When the impurity spin is "up" the interaction does not allow spin-flip for a spin-up incident electron due to conservation laws mentioned above. For this case problem reduces to that of simple impurity studied earlier as mentioned above. On the other hand when the impurity spin is "down" a spinflip scattering takes place. We compare the circulating current densities for these two cases in order to see the role of entanglement induced by the spin-flipper. Given the fact that the entanglement reduces AB-oscillations 
we study whether it also reduces the current magnification. The solid curve is for the no-flip case while the dashed one is for the spin-flip case with all other parameters kept identical. The impurity strength $(G)$ for both the cases is 4.0. In both the cases we take $l_{2} / L=0.6$ and $l_{3} / L=l_{4} / L=0.2$. The figure shows that , the circulating current for spin-flip case is significantly less than that of the no-flip case in the range $12<k L<16$. Thus one is led to believe that the flipper acting as a dephasor suppresses the quantum phenomena of current magnification.

However, this naive expectation turns out to be incorrect. This is substantiated in Fig. 3 which shows circulating current densities for the spin-flip and no-flip cases in the range $16<k L<19$ for the same lengths as mentioned above. From this figure we see that in this range of Fermi energies the amplitude of the circulating current is actually enhanced in spite of the spin-flip scattering.

Thus the flipper can not only suppress the current magnification effect but can also enhance it in some other range of Fermi energies. This effect can be ascribed to multiple reflections induced by the flipper and the junctions. The fact that entanglement due to exchange interaction between the electron and the flipper does not eliminate multiple reflections due to scattering at the impurity (and at the junctions) seems to be the reason behind the aforesaid features]. Thus far we have discussed how the flipper affects well known features of the impurity current magnification effect. However, the flipper induces some new features which we discuss below. The plot of circulating current density $\left(I_{c}\right)$ versus $k L$ for $l_{3} / L=l_{4} / L=0.25$ and $l_{2} / L=0.5$ in the range $5.6<k L<6.6$ shows an additional peak in the circulating current density arising at a point corresponding to a minimum of spin-up transmission (which is same as the maximum of the spin-down transmission). This is indicative of the spin-flip process. This effect is unique for the flipper having no counterpart in case of a simple impurity, i.e., in this region $(5.6<k L<6.2)$ no-flip scattering case does not show any circulating current. This can be ascribed to the additional phase shifts caused by spin-flip scattering along-with multiple reflections. In the range $6.2<k L<6.6$ spin-flip scattering suppresses the current magnification.

Further, we see yet another interesting feature, namely the phenomenon of current reversal. This is depicted in Fig.5. In this figure we plot the circulating current density $\left(I_{c}\right)$ versus $k L$ for $l_{3} / L=l_{4} / L=0.3125$ and $l_{2} / L=0.375$ in the wave vector range $10<k L<15$ in which we see that the spin-flip circulating current reverses its direction as compared to the no-flip case, i.e., an anti-clockwise circulating current for the no-flip case is converted into a clockwise one in the spin-flip case.

In conclusion we have shown that presence of the spinflipper which suppresses the $\mathrm{AB}$ oscillations, need not suppress the amplitude of current magnification. In fact, in certain range of Fermi energies the flipper enhances the current magnification. Apart from this it leads to current reversals and current magnification in additional Fermi energy ranges. We believe that non-suppression of this quantum effect is peculiar to elastic scattering in presence of exchange interaction leading to entanglement between the different degrees of freedom of the electron (space and spin) and the impurity spin state. This model can also be extended to study the phenomenon of quantum erasure with appropriate modifications. Only the presence of inelastic scattering, leading to irreversible loss of phase memory, can dephase $\mathrm{AB}$ oscillations and reduce current magnification simultaneously.

\section{ACKNOWLEDGMENTS}

One of us (DS) would like to thank Professor S. N. Behera for extending hospitality at the Institute of Physics, Bhubaneswar.

$\dagger$ Electronic address: colin@iopb.res.in

‡ Electronic address: joshi@iopb.res.in

* Permanent address: Materials Science Division, Indira Gandhi Center for Atomic Research, Kalpakkam 603 102, Tamil Nadu, India. Electronic address: dsahoo@iopb.res.in

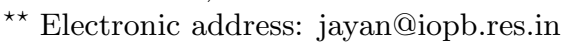

${ }^{1}$ Y. Imry, Introduction to Mesoscopic Physics (Oxford University, New York, 1997).

${ }^{2}$ S. Datta, Electronic transport in mesoscopic systems (Cambridge University Press, Cambridge, 1995).

${ }^{3}$ P. S. Deo and A. M. Jayannavar, Pramana J. Phys., (2000) in print, cond-mat/0006035.

${ }^{4}$ S. Washburn and R. A. Webb, Adv. Phys., 35 (1986) 375.

${ }^{5}$ Y. Gefen, Y. Imry, and M. Ya. Azbel, Phys. Rev. Lett. 52, 129 (1984).

${ }^{6}$ A. M. Jayannavar and P. S. Deo, Phys. Rev. B49, 13685 (1994); 51, 10175 (1995);

${ }^{7}$ P. S. Deo and A. M. Jayannavar, Phys. Rev. B50, 11629 (1994);

8 T. P. Pareek, P. S. Deo and A. M. Jayannavar, Phys. Rev. B52, 14657 (1995);

${ }^{9}$ A. M. Jayannavar and P. S. Deo, Mod. Phys. Lett. B8, 301 (1994).

10 B. C. Gupta, P. S. Deo and A. M. Jayannavar, Int. J. Mod. Phys. B10, 3595 (1996).

${ }^{11}$ M. V. Moskalets, Euro. Phys. Lett. 41, 189 (1998).

12 T. Choi, C. M. Ryu and A. M. Jayannavar, Int. J. Mod. Phys. B12, 2091 (1998); T. Choi, C. M. Ryu and A. M. Jayannavar, preprint cond-mat/9808245.

13 Sandeep.K.Joshi, Debendranath Sahoo and A. M. Jayannavar, preprint cond-mat/0007414.

${ }^{14}$ L. S. Schulman, Phys. Lett. A 211, 75 (1996).

15 A. Stern, Y. Aharonov and Y. Imry, Phys. Rev. A41, 3436 (1990).

16 J-B. Xia, Phys. Rev. B45, 3593 (1992). 
17 O. L. T. de Menezes and J. S. Helman, Am. J. Phys. 53, 1100 (1985).

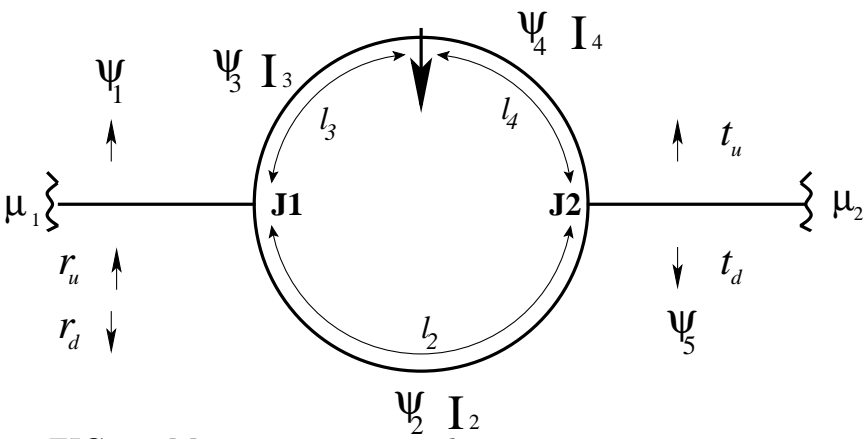

FIG. 1. Mesoscopic ring with a magnetic impurity in one arm of the ring. The situation depicted corresponds to a spin-flip scattering process.

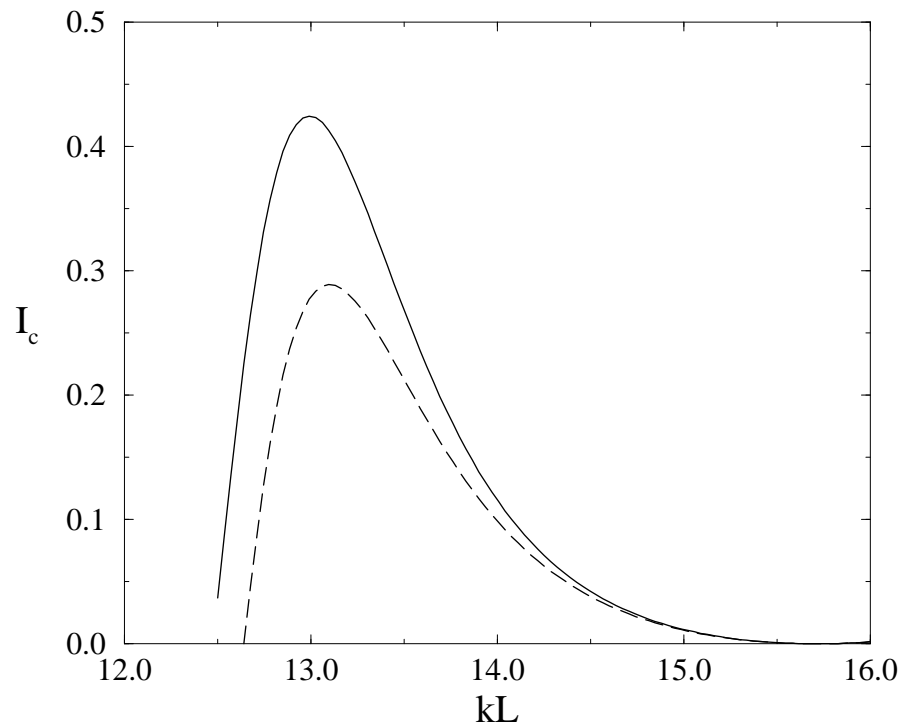

FIG. 2. Plot of circulating current density $I_{c}$ versus $k L$. $G=4.0$ and $l_{2} / L=0.6, l_{3} / L=l_{4} / L=0.2$ for both cases. The solid line is for the no-flip case while the dashed line is for the spin-flip case. This figure shows that the spin-flip process inhibits current magnification.

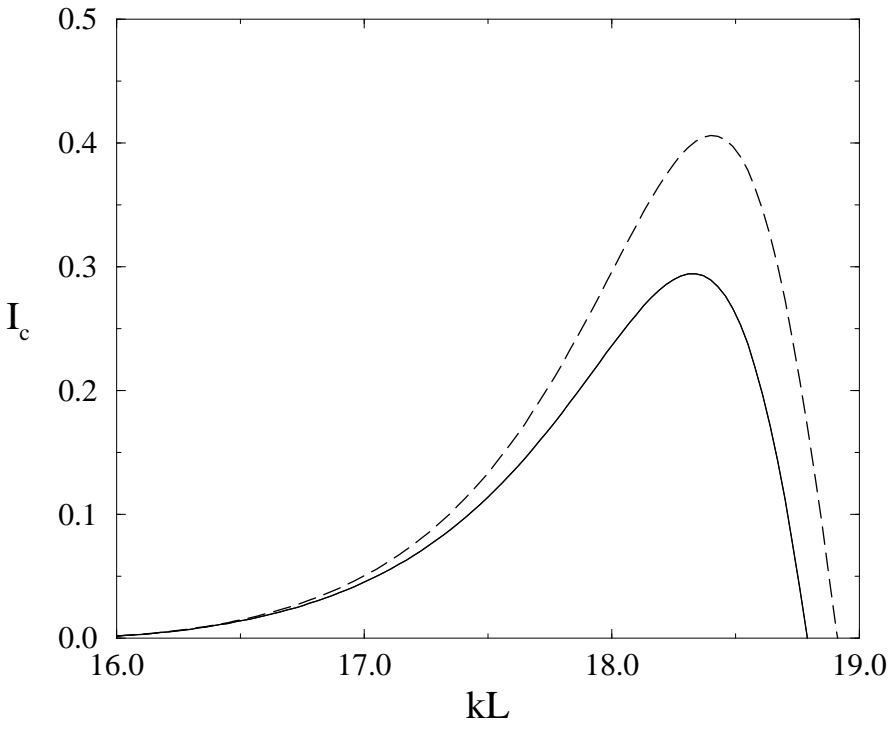

FIG. 3. Plot of circulating current density $I_{c}$ versus $k L$. $G=4.0$ and $l_{2} / L=0.6, l_{3} / L=l_{4} / L=0.2$ for both cases. The solid line is for the no-flip case while the dashed line is for the spin-flip case. This figure in contrast to Fig. 2 shows that the spin-flip process enhances current magnification.

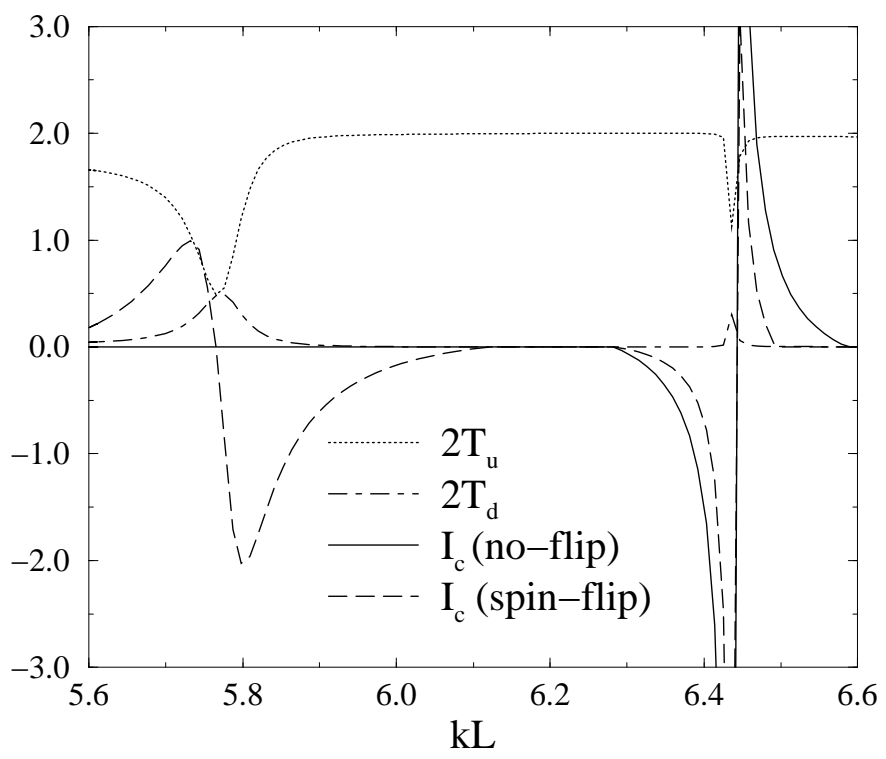

FIG. 4. Plot of circulating current density $I_{c}$ versus $k L$. $G=4.0$ and $l_{2} / L=0.5, l_{3} / L=l_{4} / L=0.25$ for both cases. The solid line is for the no-flip case while the dashed line is for the spin-flip case.The dash-dotted line is for $2 T_{d}$ while the dotted line is for $2 T_{u}$ wherein $T_{u}=\left|t_{u}\right|^{2}$ and $T_{d}=\left|t_{d}\right|^{2}$. 


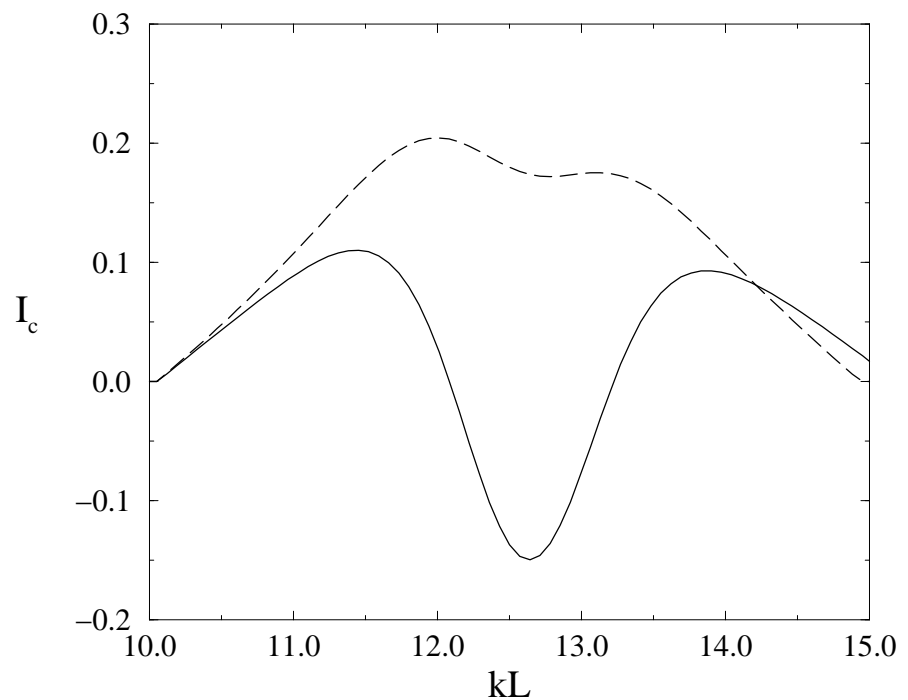

FIG. 5. Plot of circulating current density $I_{c}$ versus $k L$. $G=4.0$ and $l_{2} / L=0.375, l_{3} / L=l_{4} / L=0.3125$ for both cases. The solid line is for the no-flip case while the dashed line is for the spin-flip case. 